\title{
Curvature Sensor Based on a Long-Period Grating in a Fiber Ring Resonator Interrogated by an OTDR
}

\author{
Regina MAGALHÃES, Susana SILVA, and Orlando FRAZÃO* \\ INESC TEC and Department of Physics and Astronomy, Faculty of Sciences of University of Porto, Porto 4169-007, \\ Portugal \\ *Corresponding author: Orlando FRAZÃO E-mail: ofrazao@inesctec.pt
}

\begin{abstract}
The proposed technique demonstrates a fiber ring resonator interrogated by an optical time domain reflectometer (OTDR), for intensity sensing. By using this methodology, a cavity round trip time of $2.85 \mu \mathrm{s}$ was obtained. For a proof of concept, a long-period grating was inserted in the resonant cavity operating as a curvature sensing device. A novel signal processing approach was outlined, regarding to the logarithmic behavior of the OTDR. Through analyzing the experimental results, an increase in the measured sensitivities was obtained by increasing applied bending. With curvatures performed from $1.8 \mathrm{~m}^{-1}$ to $4.5 \mathrm{~m}^{-1}$, the sensitivity values ranged from $2.94 \mathrm{~dB} \cdot \mathrm{km}^{-1}$ to $5.15 \mathrm{~dB} \cdot \mathrm{km}^{-1}$. In its turn, the sensitivities obtained presented a linear behavior when studied as a function of the applied curvature, following a slope of $0.86 \times 10^{-3} \mathrm{~dB}$. The advantages of applying this technique were also discussed, demonstrating two similar fiber rings multiplexed in a series of configurations.
\end{abstract}

Keywords: Cavity ring-down; curvature; fiber ring resonator; long period grating; optical fiber sensors; OTDR

Citation: Regina MAGALHÃES, Susana SILVA, and Orlando FRAZÃO, "Curvature Sensor Based on a Long-Period Grating in a Fiber Ring Resonator Interrogated by an OTDR,” Photonic Sensors, 2020, 10(1): 1-6.

\section{Introduction}

Fiber ring resonators operating with optical time domain reflectometer (OTDR) have been largely used in the last decades in very different configurations. This technique was firstly demonstrated in 1992 describing a reduction in fading noise which caused an amplitude fluctuation on a backscattered trace in a coherent OTDR [1], and the characteristics of fading noise in Rayleigh backscattering measurements made with coherent light waves such as in coherent-OTDR and coherent-OFDR were also studied [2].

A new method for characterization of optical fiber resonators using an OTDR technique was reported in 1997 [3]. This technique yielded accurate results for cavity lengths ranging from a few meters to several kilometers, with the advantage of being easy to implement. Later in that year, a fiber-optic gyroscope based on Rayleigh backscattering in a fiber-ring resonator was demonstrated [4]. The combination of two-ring-resonator schemes of the fiber optic rotation sensor was developed and demonstrated in 2007 [5]. The two-ring topology structure was studied with two rings in series and in parallel.

The OTDR can be implemented by interrogating a very wide range of sensors, including Fabry-Pérot

Received: 1 January 2018 / Revised: 30 March 2018

(C) The Author(s) 2019. This article is published with open access at Springerlink.com DOI: $10.1007 / \mathrm{s} 13320-019-0398-3$

Article type: Regular 
interferometer [6], long-period gratings (LPG) [7], singlemode-multimode-singlemode sensors [8], and fiber Bragg gratings [9]. In the first case, interrogating a Fabry-Pérot, the results present a much more similar spectrum comparing with the ones obtained by using a fiber ring resonator.

This work proposes a main setup composed by a fiber ring resonator interrogated by an OTDR.
For a proof of concept, an LPG was inserted inside the fiber ring to operate as an intensity sensor. This resonating cavity was interrogated by the OTDR, as the curvature on the sensor was applied.

\section{Experimental results}

The experimental setup of the proposed system is presented in Fig. 1.

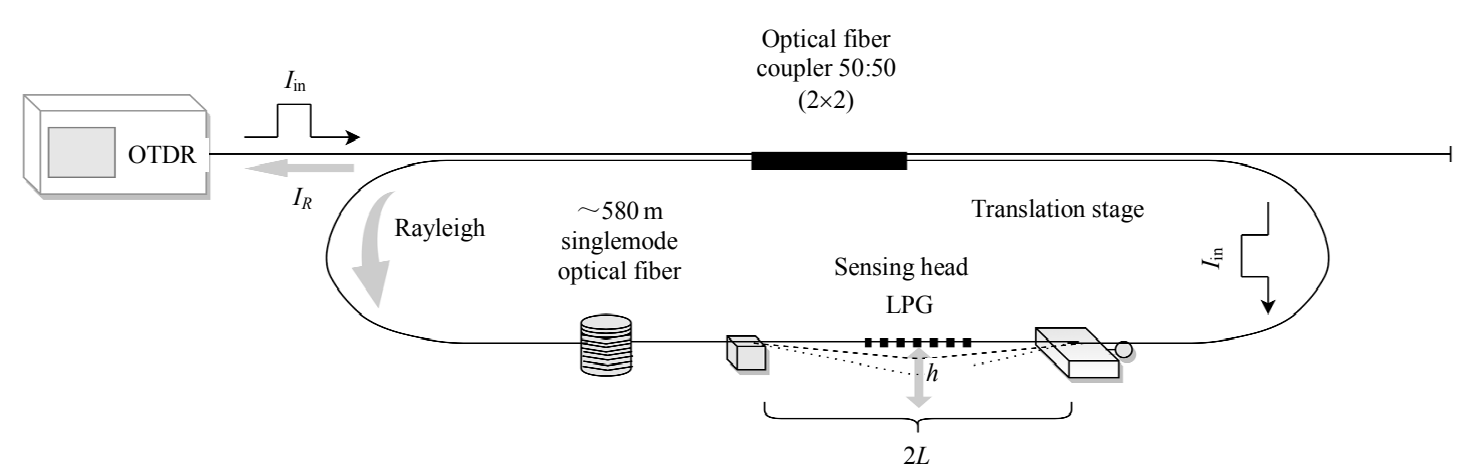

Fig. 1 Curvature sensor measuring setup - the signal is introduced by the OTDR, which uses the backscattered light to make sensitivity measurements.

The main setup is composed by an OTDR operating at $1550 \mathrm{~nm}$, used as a modulated multimode laser transmitter to send a square pulse of light (50 ns) down into a singlemode optical fiber (SMF). The output port 4 was connected to the input port 3 through a 3-dB-singlemode fiber coupler $(2 \times 2)$, in order to form a fiber ring resonator (see Fig. 1). This fiber loop with $\sim 580 \mathrm{~m}$ was placed at a very short distance from the OTDR, allowing the use of the backscattered light detection to make measurements. An LPG with a central wavelength at $1559 \mathrm{~nm}$, a resonance with $-4.70 \mathrm{~dB}$ loss, and a period of $540 \mu \mathrm{m}$ was also used. The LPG was inserted into the setup, more precisely inside the fiber ring resonator, rather than that being fixed to an optical fiber translation stage. This setup formed the proposed curvature sensor measuring system. When the LPG is submitted to curvature, it undergoes wavelength and intensity variations. However, in this case, the LPG was used only as an intensity sensor.

An important feature of the fiber ring resonator is that the pulse $I_{\text {in }}$ circulates several times inside the ring (see Fig. 1). After entering the resonator, 50\% of the light remained circulating inside the ring, while the other $50 \%$ traveled to the point at which the fiber ends. At the same time, Rayleigh backscattered light was formed inside of the fiber loop, and, in turn, it also underwent several passages through the ring in the opposite direction [4]. Afterwards, the backscattered light and reflected light were sent back in direction to the OTDR $\left(I_{R}\right)$ through the fiber coupler. The result is a set of consecutive peaks separated by a distance that corresponds to the cavity length. The first pulse was coupled out via the $50 \%$ arm of the output port without circulating around the fiber loop. The remaining ones, which underwent an amplitude decay with time were originated by the total existing losses in the fiber loop, such as fiber couplers insertion losses, fiber loss, and LPG transmission attenuation, being related to the consecutive passages of light through the fiber resonator.

The pulse width was adjusted in order to 
optimize the distance measurements. Nonetheless, it was necessary to take into account that an increase in the power of pulses created a larger backscattered signal, but also led to losses of resolution between events. Despite the fact that the larger pulses led to traces with better performances in terms of distance capability, and also provided features with less noise, the capability to identify events was compromised. This capability decreased since the test pulse overloaded the OTDR, reducing its ability to see events in the neighborhood. In this case, to obtain better noise performances, the pulse width was set at $50 \mathrm{~ns}$, which was the width that allowed a better balance between the situations previously described, corresponding to the optimal width studied for the proposed configuration.

\subsection{Processing technique}

The profile shown in the OTDR can be seen in Fig. 2. The intensity was measured $(\mathrm{dB})$ in detriment of the distance travelled by light. This behavior was also studied as a linear function, resulting in several peaks with an exponential profile (trace fitting was performed), as can be seen in the graphic inset of Fig. 2.

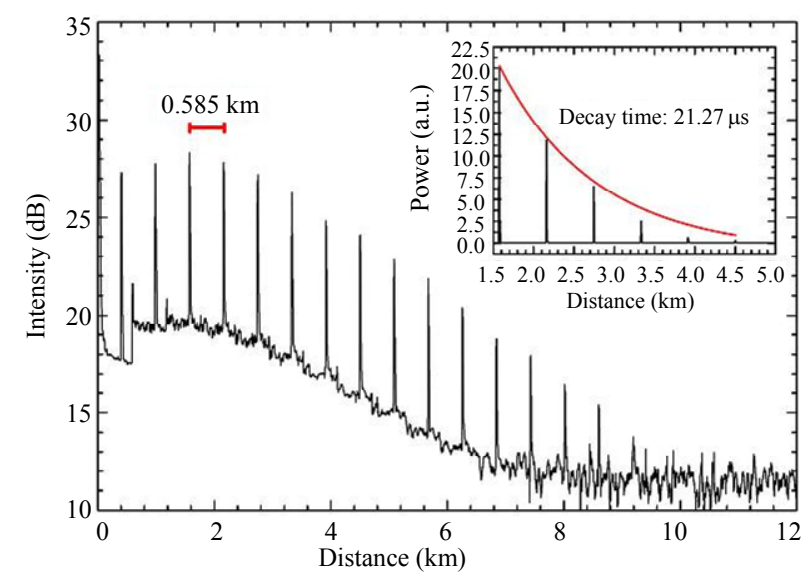

Fig. 2 Intensity as a function of the length along the fiber, representing the OTDR profile for the absence of applied curvature. Inset is the linear behavior of the OTDR.

The peaks shown in Fig. 2 are spaced from $0.585 \mathrm{~km}$, which corresponds to the length of the optical fiber used in this fiber resonator, and a cavity round trip time of $2.85 \mu$ s was achieved.

Each peak was resulted from the addition of light contributions circulating in the ring for a given propagation time, i.e., the light that remained circulating inside the ring and also the Rayleigh backscattered light that also occurred inside it [3].

The trace in the OTDR presents an increasing intensity behavior for the first set of peaks, given by a weak contribution of backscattered light (see Fig. 2). The preceding peaks present a decreasing intensity behavior due to an increasing in Rayleigh backscattered light and increasing intrinsic losses of the ring.

\subsection{Curvature measurements}

The behavior of this structure as a curvature sensor was characterized. The fiber was fixed at two points, at a distance of $104 \mathrm{~mm}$ from each other $(2 L)$. One of the fixed points coincides with a translation stage, which, in turn, allowed the appliance of curvature on the fiber. The bending displacement, $h$, was applied to the LPG via sequential $1 \mathrm{~mm}$ displacements. The change in the curvature was measured according to $2 h /\left(h^{2}+L^{2}\right)$ [10], where $L$ was the half distance between the two fixed points.

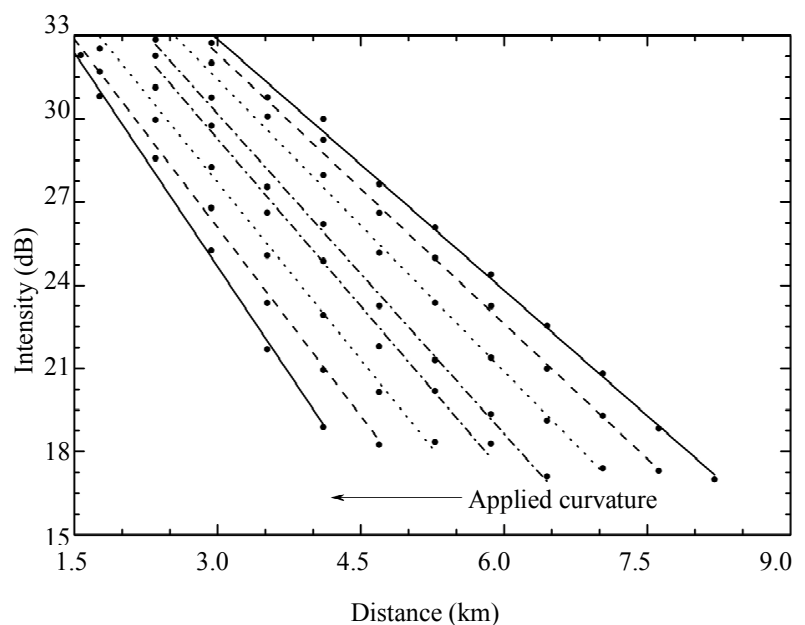

Fig. 3 Linear fits performed to the data varying with the applied curvature.

Through analyzing the logarithmic signal of the OTDR (see Fig. 3), a decrease in the peaks appeared according to a linear tendency which follows a 
certain slope. In turn, this slope was associated with the sensitivity obtained by measuring the amplitude variation of the output peaks when applying curvature on the LPG. The different tendencies registered are represented in Fig. 3, for curvature applied from $1.8 \mathrm{~m}^{-1}$ to $4.5 \mathrm{~m}^{-1}$.

Directly from Fig. 3, one can observe a pattern in the measurements, since the data shows an increasing slope with increasing applied curvature. Bending the LPG induces losses in the ring results in an increase in the decay time, represented by the increasing slopes. Concerning the results obtained from Fig. 3, the absolute value of the different slopes are obtained, representing the sensitivities achieved, ranged from $(2.94 \pm 0.05) \mathrm{dB} \cdot \mathrm{km}^{-1}$ to $(5.15 \pm 0.05) \mathrm{dB} \cdot \mathrm{km}^{-1}$.

Figure 4 shows an analysis of the behavior of the sensitivity according to applying curvature, being the data extracted directly from Fig. 3. Therefore, by performing four linear fits to the data, an analysis regarding the variation of the intensity over curvature can be obtained. This also allows to understand the behavior of the intensity of the system in terms of the radius of curvature $\left(\mathrm{m}^{-1}\right)$.

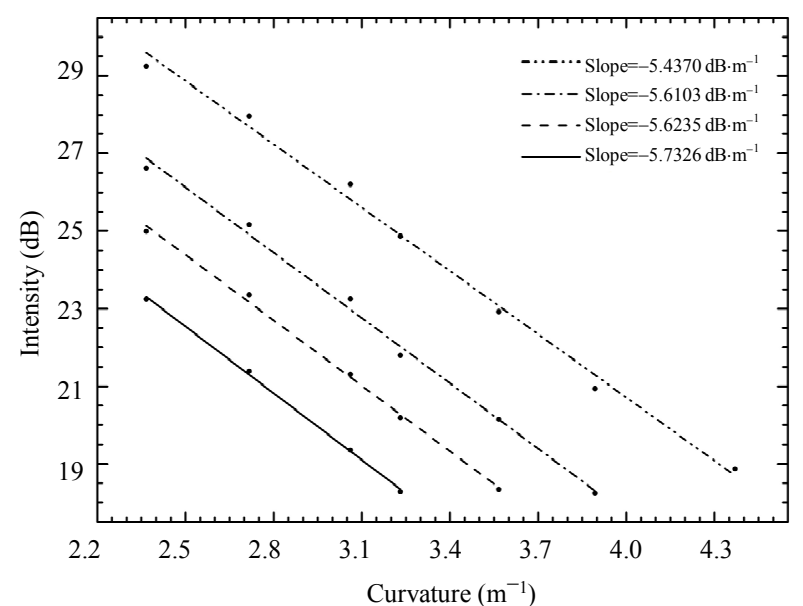

Fig. 4 Sensitivities obtained in the OTDR along the distance measurements.

The results show a behavior characterized by increasing sensitivities with increasing distances.

Figure 5 represents the relationship between different sensitivities depicted and different applied curvature values. From these results, a linear increase in the measurements sensitivity with increasing applied curvature was registered. The relation between the sensitivities achieved and the applied bending presented a linear behavior (a linear fit was performed to the data), which followed a slope of $(0.86 \pm 0.02) \times 10^{-3} \mathrm{~dB}$.

When comparing this work with the one in [7], where an LPG was interrogated directly by an OTDR, one advantage is obtained, i.e., a large bandwidth of curvature measurement could be achieved with the proposed processing signal. Also, no saturation regime was measured and, considering results from Fig. 4, a mean sensitivity of $5.5 \mathrm{~dB} / \mathrm{m}^{-1}$ was obtained, for the range from $3.3 \mathrm{~m}^{-1}$ to $3.8 \mathrm{~m}^{-1}$ of applied curvature, which corresponded to about double of the sensitivity obtained in [7].

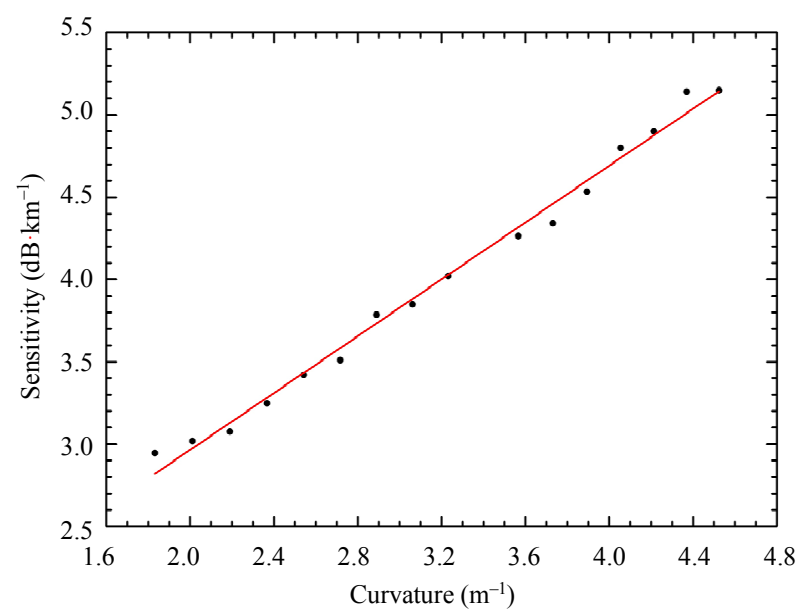

Fig. 5 Sensitivities measured in the OTDR along the applied curvature.

Another advantage of using the OTDR is the attainment of multiplexed signals when implementing a configuration with two or more fiber ring loops in series. The presented configuration in Fig. 6 shows an example of this type of setup, with two fiber rings in series, separated by $20 \mathrm{~km}$ of fiber. The first ring has a 580-m-long fiber loop while the second is 250 -m-long.

Figure 7 shows the respective trace obtained by the OTDR, where the two signals are multiplexed and perfectly differentiated. The signal with respect to the first fiber ring presents a cavity round trip 
time of $2.84 \mu \mathrm{s}$, as expected from the previously proposed experiment (see Fig. 2), while the second fiber ring presents a cavity round trip time of $1.26 \mu \mathrm{s}$.

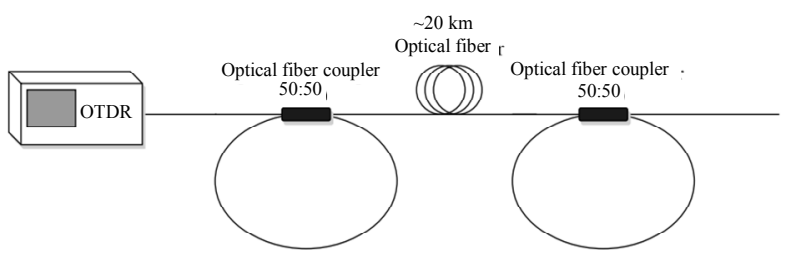

Fig. 6 Experimental setup using two fiber rings in series, directly interrogated by an OTDR.

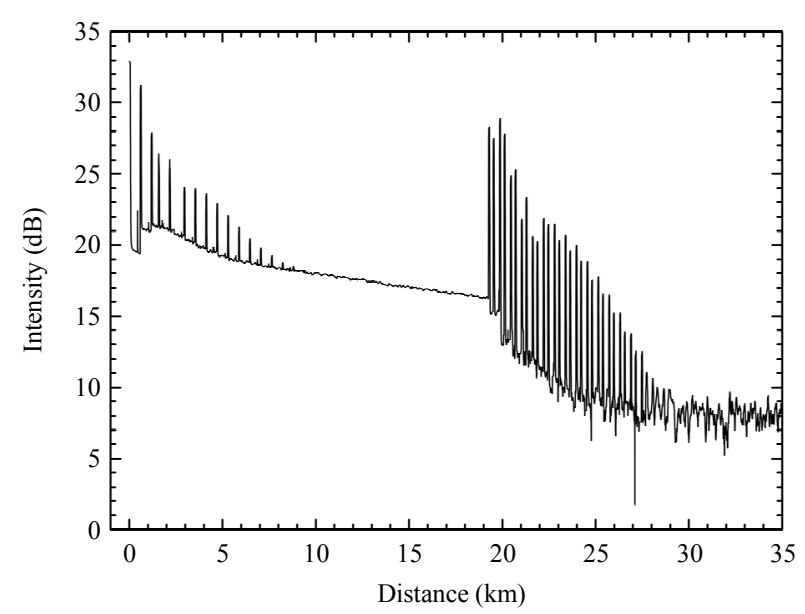

Fig. 7 OTDR trace obtained from the two rings in series configuration, representing the multiplexed signals.

The ring down value of the second fiber ring is independent of the signal provided by the first fiber loop. Since this separation does not occur using the usual interrogation system composed by a photodetector and an oscilloscope, this setup can be significantly useful in more complex systems.

\section{Conclusions}

A curvature sensing configuration was proposed by using a fiber ring resonator interrogated by an OTDR. It was observed that this type of interrogation is possible, and also that the depicted response is similar to the one obtained in a cavity ring-down configuration, studying the linear behavior of the OTDR.

Through analyzing the trace of the OTDR, a cavity round trip time of $2.84 \mu$ s was measured. The cavity round trip time achieved corresponds to a value obtained by using backscattered light to interrogate the fiber ring resonator. Nonetheless, if an interrogation technique composed by a photodetector and an oscilloscope (very commonly used in the cavity ring-down configuration) was used, the cavity round trip time registered had to be the same for this setup.

As a proof of concept, an LPG was inserted in this system, operating as an intensity sensor. The obtained results show an increase in the sensitivities measured with increasing implemented bending to the fiber. With applied curvatures varying from $1.8 \mathrm{~m}^{-1}$ to $4.5 \mathrm{~m}^{-1}$, the sensitivity values achieved ranged from $(2.94 \pm 0.05) \quad \mathrm{dB} \cdot \mathrm{km}^{-1}$ to $(5.15 \pm 0.05) \mathrm{dB} \cdot \mathrm{km}^{-1}$. The measured sensitivities presented a linear behavior when studied as a function of the applied curvature, following a slope of $(0.86 \pm 0.02) \times 10^{-3} \mathrm{~dB}$. This configuration can be easily be implemented in a more complex one, using multiplexed resonators, since it allows a much more easy data procession technique by presenting separated signals. However, when inserting intensity sensors, the length of the resonators and the distance between sensors must be considered in order to obtain satisfying results. This type of configuration can be easily implemented in suspended electrical power cables for measurements of the catenary curve.

\section{Acknowledgment}

This work is financed by National Funds through the Portuguese funding agency, FCT - "Fundação para a Ciência e a Tecnologia" under Grant No. UID/EEA/50014/2019.

Open Access This article is distributed under the terms of the Creative Commons Attribution 4.0 International License (http://creativecommons.org/licenses/by/4.0/), which permits unrestricted use, distribution, and reproduction in any medium, provided you give appropriate credit to the original author(s) and the source, provide a link to the Creative Commons license, and indicate if changes were made. 


\section{References}

[1] H. Izumita, S. I. Furukawa, Y. Koyamada, and I. Sankawa, "Fading noise reduction in coherent OTDR," IEEE Photonics Technology Letters, 1992, 4(2): 201-203.

[2] K. Shimizu, T. Horiguchi, and Y. Koyamada, "Characteristics and reduction of coherent fading noise in Rayleigh backscattering measurement for optical fibers and components," Journal of Lightwave Technology, 1992, 10(7): 982-987.

[3] A. Küng, J. Budin, L. Thévenaz, and P. A. Robert, "Optical fiber ring resonator characterization by optical time-domain reflectometry," Optics Letters, 1997, 22(2): 90-92.

[4] A. Küng, J. Budin, L. Thévenaz, and P. A. Robert, "Rayleigh fiber optics gyroscope," IEEE Photonics Technology Letters, 1997, 9(7): 973.

[5] L. B. Yuan, Z. H. Liu, and J. Yang, "Rayleigh backscattering fiber optic rotation sensor based on combined two-ring-resonator," Sensors and Actuators A: Physical, 2007, 136(1): 216-220.

[6] M. Giraldi, C. Fernandes, M. Ferreira, M. J. de Sousa, P. Jorge, J. Costa, et al., "Fiber loop mirror sensors interrogated and multiplexed by OTDR", Journal of Lightwave Technology, 2015, 33(12): 2580-2584.

[7] O. Frazão, R. Falate, J. M. Baptista, J. L. Fabris, and J. L. Santos, "Optical bend sensor based on a long-period fiber grating monitored by an optical time-domain reflectometer," Optical Engineering, 2005, 44(11): 110502-110502.

[8] M. Giraldi, C. Fernandes, M. S. Ferreira, M. J. de Sousa, P. Jorge, J. C. Costa, et al., "Fiber optic displacement sensor based on a double-reflecting OTDR technique," Microwave and Optical Technology Letters, 2015, 57(6): 1312-1315.

[9] A. D. Kersey, M. A. Davis, and T. Tsai, "Fiber optic Bragg grating strain sensor with direct reflectometric interrogation," in Proceeding of Eleventh International Conference on Optical Fiber Sensors, Sapporo, Japan, 1996, pp: Th45.

[10] S. Silva, E. G. P. Pachon, M. A. R. Franco, P. Jorge, J. L. Santos, F. X. Malcata, et al., "Curvature and temperature discrimination using multimode interference fiber optic structures-a proof of concept," Journal of Lightwave Technology, 2012, 30(23): 3569-3575. 